\title{
Changing Beliefs about Trauma: A Qualitative Study of Cognitive Processing Therapy
}

\author{
Jennifer L. Price \\ Georgetown College, Georgetown, USA \\ Helen Z. MacDonald \\ Emmanuel College, Boston, USA \\ Kathryn C. Adair \\ University of North Carolina at Chapel Hill, USA \\ Naomi Koerner and Candice M. Monson \\ Ryerson University, Toronto, Canada
}

\begin{abstract}
Background: Controlled qualitative methods complement quantitative treatment outcome research and enable a more thorough understanding of the effects of therapy and the suspected mechanisms of action. Aims: Thematic analyses were used to examine outcomes of cognitive processing therapy (CPT) for posttraumatic stress disorder (PTSD) in a randomized controlled trial of individuals diagnosed with military-related PTSD $(n=15)$. Method: After sessions 1 and 11, participants wrote "impact statements" describing their appraisals of their trauma and beliefs potentially impacted by traumatic events. Trained raters coded each of these statements using a thematic coding scheme. Results: An analysis of thematic coding revealed positive changes over the course of therapy in participants' perspective on their trauma and their future, supporting the purported mechanisms of CPT. Conclusion: Implications of this research for theory and clinical practice are discussed.
\end{abstract}

Keywords: Cognitive therapy, treatment outcome, qualitative methods, PTSD.

\section{Introduction}

Randomized controlled trials demonstrate the efficacy of cognitive processing therapy (CPT; Resick, Monson and Chard, 2007) for posttraumatic stress disorder (PTSD) (e.g. Chard, 2005; Resick et al., 2008). CPT reduces military-related PTSD symptoms, as well as co-occurring

Reprint requests to Jennifer L. Price, Associate Professor of Psychology, Georgetown College, 400 E. College Street, Georgetown, KY 40324, USA. E-mail: jennifer_price@georgetowncollege.edu 
depression and general anxiety symptoms, while simultaneously improving affect regulation and social functioning (Monson et al., 2006). However, self-report instruments and other quantitative measures are incomplete in their ability to fully capture the effects of therapy and the putative mechanisms of change (e.g. Gallegos, 2005). Despite a call for psychotherapy outcome research that incorporates qualitative assessments of outcomes and hypothesized mechanisms (Klein and Elliott, 2006), few studies have implemented this pluralistic approach in PTSD treatment research.

Studies employing a thematic approach in analyzing trauma narratives have largely focused on specific elements of the narrative, such as the degree of fragmentation or disorganization of the trauma memory (see O'Kearney and Perrott, 2006, for a review), and then have correlated these elements with treatment response. For example, Klest and Freyd (2007) created a global rating scale that examined the coherence and cohesion of participants' trauma narratives and found that better organized narratives at pretreatment predicted decreases in physical and mental health symptoms at posttreatment.

Shearing, Lee and Clohessy (2011) studied patients' experiences of reliving aspects of the trauma memory within the context of trauma-focused cognitive-behavioral therapy (TFCBT), whereas Vincent, Jenkins, Larkin and Clohessy (2012) investigated the effectiveness and appropriateness of TF-CBT for asylum-seekers. Shearing and colleagues (2011) found that although reliving the trauma was reported to be challenging and painful, participants described the experience as achievable and rewarding (Shearing et al., 2011). Vincent and colleagues (2012) identified particular experiences that encouraged (e.g. trusting the therapist) and impeded (e.g. difficulty coping with exposure exercises) patients' engagement in therapy.

Specific to CPT, Sobel, Resick and Rabalais (2009) investigated changes in "impact statements" that individuals write after the first session and just prior to the final session about their perception of the impact of their trauma. The first impact statement is used to assess trauma-related beliefs that may impede recovery, and the latter impact statement is used to document changes in thinking that may occur during therapy. Consistent with the cognitive theory underlying CPT, Sobel and colleagues coded the statements for accommodation (i.e. healthy modification of existing schemas in a balanced and accurate way - "I did the best I could to protect myself"), assimilation (i.e. misappraising the trauma to be consistent with preexisting schema - "I should have been able to stop it from happening"), or over-accommodation (i.e. overgeneralized changes to preexisting schema "All men are dangerous"). Final impact statements included a significantly higher percentage of accommodated clauses and a significantly lower percentage of assimilated and overaccommodated clauses compared with initial statements. At posttreatment, self-reported PTSD symptoms were positively associated with over-accommodation clauses and negatively associated with accommodation, suggesting that the suspected cognitive processes largely corresponded with response to treatment.

In the current analysis, qualitative methods were used to examine participants' appraisals of their trauma experiences to gain a more complete understanding of the outcomes and purported mechanisms of CPT. We considered using a computerized text analysis program (e.g. Linguistic Inquiry Word Count; Pennebaker, Booth and Francis, 2007; General Inquirer; Stone, 1997) to apply quantitative strategies to our linguistic data. However, one important limitation of computerized text analysis is its inattention to context and meaning of clauses due to its focus on word counts; miscoding of meaning can occur when words are examined in isolation from the context in which they are embedded (Tausczik and Pennebaker, 2010). In 
our study, taking emotion words out of context often meant losing the valence of the statement. For example, after receiving CPT, one patient wrote, "I am less irritable", which would be coded as a negative emotion by linguistic computer software because of the word "irritable", although the statement was clearly positively-valenced. Therefore, we chose to examine our data using thematic analysis. In thematic analysis, researchers use a theoretically- and empirically-derived coding system to examine participants' language and the corresponding themes that emerge. Thematic analysis minimizes the risk of misinterpretations and can be used to capture more complex changes in thought processes, such as shifts in perspective.

This investigation examined therapeutic outcomes and mechanisms of action in CPT using thematic analysis of patients' initial and final impact statements. Exploratory aims were to: (i) Investigate how patients' perspectives on CPT themes of safety, trust, power/control, esteem, and intimacy might change from pre to posttreatment; and (ii) Examine whether other themes would emerge in either initial or final statements. Given existing treatment outcome data and the purported mechanisms of action for CPT, we hypothesized that patients would articulate healthier cognitions in the final impact statement compared with the initial impact statement.

\section{Method}

\section{Participants}

The current study focused on a subset of participants from a randomized controlled waitlist trial of 60 individuals diagnosed with military-related PTSD (Monson et al., 2006). PTSD diagnoses were established using the Clinician Administered PTSD Scale (CAPS; Blake et al., 1995). Participants included those who were immediately randomized to 12 sessions of CPT (see Resick et al., 2007 for a detailed description of CPT) and who had complete impact statement data available $(n=15)$. These participants' demographic and clinical characteristics were consistent with those of the original sample: predominately male (87\%), married $(67 \%)$, Vietnam war veterans $(80 \%)$, with a mean age of $52.87(S D=6.61)$ years. Severity of PTSD and depression at pretreatment were not statistically different from the overall sample (e.g. CAPS $M=81.33$, BDI $M=26.87$ ).

\section{Procedure}

The directions for writing the impact statements were: "Please write at least one page on what it means to you that this traumatic event occurred. Consider the effects this traumatic event has had on your beliefs about yourself, others, and the world. Also, consider the following topics while writing your answer: safety, trust, power/control, esteem, and intimacy." Consistent with the CPT protocol, initial impact statements were assigned at the conclusion of session 1; final impact statements were assigned at the conclusion of session 11.

\section{Thematic analysis}

Blinded to time of writing, authors HZM and KCA coded the impact statements by identifying every individual thought unit in each statement. We defined a thought unit as a section of text that contained one main idea. Thought units were often the length of a full sentence (e.g. "I will never trust authority without question again"), but were sometimes clauses within 
sentences conveying more than one idea (e.g. "I feel as if the world is not safe and that I shouldn't trust anyone" consists of two thought units). Raters achieved a high level of interrater reliability in identifying thought units (94.6\% agreement); discrepancies were resolved by reaching consensus among the two coders and the first author.

An inductive method of categorizing thought units was used. First, a list of thought units for each patient was developed. Then, these thought units were organized into subthemes across all participants according to similarity of content (e.g. "I have to be the one in control and have all the power" and "I feel I need to control everything and keep the upper hand at all times" were both classified into the subtheme "Efforts to control too much"). These subthemes were then organized into larger overarching themes (e.g. "Efforts to control too much" is one of three subthemes within the overarching "Power" theme; see Table 1). Subthemes from initial impact statements were compared with subthemes from final impact statements to examine changes in language that might be attributed to treatment. Quotations provided below were extracted from patient narratives and are presented here as exemplars of the thought units/subthemes most commonly conveyed in the impact statements. To maintain confidentiality, we randomly assigned each patient $(\mathrm{P})$ a number (1-15) and indicated whether the quotation was from the patient's initial (i) or final (f) impact statement.

\section{Results}

Analyses resulted in 425 thought units in initial impact statements and 391 thought units in final impact statements, totaling 816 thought units. Thirty-four thought units were excluded either because the contents of the thoughts were unclear, or because the patient did not follow the assignment instructions. The resulting 782 thought units were grouped into 56 subthemes, 9 of which were excluded from analyses due to low frequency (i.e. fewer than 4 thought units). The remaining 47 subthemes were then inductively collapsed into 9 overarching themes. As anticipated, five of the overarching themes corresponded with the major themes of CPT, which patients were directed to address in the assignment: safety, trust, power/control, esteem, and intimacy. The four other overarching themes that emerged were emotions/symptoms, perspective on important life issues, education/work, and positive effects of therapy.

\section{Safety}

The most common safety subtheme in the initial impact statements was the feeling of being unsafe. Participants often described isolating themselves as a mechanism to ensure their safety:

P15i: I feel there is no safe place in this world anymore. The only place I feel safe is out in the woods with no one around. But then there are times that isn't safe.

In one statement, a patient's overwhelming difficulties following trauma exposure resulted in a disregard for his own safety:

P2i: As far as safety I don't care anymore sometime[s] I wish it was over with.

In their final impact statements, some patients made strong claims regarding new found feelings of safety: 
Table 1. Themes and sub-themes expressed in impact statements

\begin{tabular}{|c|c|}
\hline Theme & Sub-theme \\
\hline \multirow[t]{4}{*}{ Safety } & Fighting for life/survival \\
\hline & No consideration/responsibility for safety \\
\hline & Feel unsafe \\
\hline & Feeling safe/adopting a more balanced view of safety \\
\hline \multirow[t]{4}{*}{ Trust } & Lack of trust in others \\
\hline & Lack of trust in institutions/authority figures \\
\hline & Trust in oneself \\
\hline & Trusting others \\
\hline \multirow[t]{3}{*}{ Power/control } & Powerless \\
\hline & Efforts to control too much \\
\hline & Diminished need to over-control \\
\hline \multirow{5}{*}{ Esteem } & Lack of self-esteem \\
\hline & Feel pride/good about self \\
\hline & Not supported/respected by others \\
\hline & Recognizing that patient did all that s/he could in the situation \\
\hline & Self-blame \\
\hline \multirow[t]{5}{*}{ Intimacy } & Lack of intimacy \\
\hline & Problems with sexual intimacy \\
\hline & Problems in specific relationships \\
\hline & Changes in thoughts/experiences about/of intimacy \\
\hline & Experience closeness with others \\
\hline \multirow[t]{10}{*}{ Emotions/symptoms } & Emotional numbing/lack of positive affect \\
\hline & Experience of general negative affect \\
\hline & Experience of guilt \\
\hline & Not feeling guilty \\
\hline & Experience of fear \\
\hline & Experience of anger \\
\hline & Experience of sadness \\
\hline & Experience of happiness and other positive emotions \\
\hline & PTSD symptoms \\
\hline & Reference to alcohol/drug use \\
\hline \multirow[t]{9}{*}{ Perspective } & Trauma changed my outlook/perspective (general) \\
\hline & Became pessimistic \\
\hline & Suspicious of institutions \\
\hline & Experience of regrets \\
\hline & New perspective of actions taken during trauma \\
\hline & Desire change \\
\hline & My perspective has changed for the better \\
\hline & Trauma changed me negatively \\
\hline & Trauma taught me something about the world/living \\
\hline \multirow[t]{2}{*}{ Education/work } & Negative references to education/work \\
\hline & Military life \\
\hline \multirow[t]{5}{*}{ Positive effects of therapy } & Positive effects on esteem \\
\hline & Positive effects on thought processes \\
\hline & Grateful to therapist/referring doctor/therapy itself \\
\hline & Therapy was challenging \\
\hline & Not "cured"/expect to continue to improve \\
\hline
\end{tabular}


P10f: ... I feel safer because I am active and not helpless anymore.

Others' claims were more moderated at the conclusion of therapy, but still included concerns about safety:

P5f: I feel a little safer around. I am safer [than I] would allow myself before.

\section{Trust}

Initial impact statements were characterized by patients' lack of trust in others, as well as feeling unsafe and worrying about being hurt again:

P8i: I have little trust in others as I feel that most people will get to know me and not like me and they will try to do something else bad to me.

P12i: I have a lack of trust for people that are in charge and look for reasons to confirm that lack of trust. If someone is in charge and shows in any way that they are not to be trusted, I get angry.

Patients' mistrust was frequently centered on a specific person or organization. Commonly, patients described a mistrust of governmental representatives or agencies:

P3i: I have come to distrust authority at the local, state, and especially the federal levels ... I have carried a grudge, felt unsafe, and have come to mistrust the government ... for so long.

In contrast, in final impact statements, patients described an increased ability to trust themselves and others. Patients' newfound trust was often associated with an increased sense of safety:

P6f: I realize now that I can trust my judgment of people and can protect myself as best as I can each step of the way as I get to know them...I also do not look at all people as being harmful and out to get me.

Some patients described improvements in trust, but acknowledged continued difficulty after treatment:

P2f: Trust for me is very hard people need to prove to me that I can trust them, not that I can trust them until they do something wrong.

P4f: I trust people more - to a small degree.

\section{Power/control}

In the initial impact statements, many patients reported believing they lacked control over their lives. One patient described how this feeling began following his exposure to war:

P9i: [Vietnam] taught me that death is everywhere, instant and non-negotiable. Nam took away any chance of "running away". In a very real sense I am much more vulnerable to attack now than before. Physically I cannot run, so psychically I cannot run either.

Whereas some patients' initial impact statements described a complete lack of control, others articulated attempts to control every aspect of their environment: 
P7i: I have to be the one in control and have all power. I am scared to death that if some other family member takes control something bad will happen to me.

In the final impact statements, many patients had developed a more balanced view of power and control, as represented by the following statement:

P9f: Also, my attempts at "overcontrol" of every single situation I encounter are now diminishing. I need not control every situation to live. People will do what they will. I need not micro-manage my environment at all times.

\section{Esteem}

In their initial impact statements, patients commonly described the trauma causing a loss of self-esteem. Patients blamed themselves for aspects of the trauma, as well as for other, unrelated, life events that occurred subsequent to their traumas:

P6i: I feel that because of what took place with [my husband] that there is something wrong with me and the way that I interact with people. That I bring out the worst in people... I don't like myself ... if I was a better person or more of a woman then none of this would have happened to me... I am afraid to show who I am because I feel I am very unworthy.

In the final impact statements, patients described improved self-esteem related to the trauma and in other domains of life. They often spoke about pride in themselves and in decisions they made during and following the trauma, while others were more measured in their responses:

P9f: My self-worth has increased during therapy. I no longer look on myself as fundamentally flawed. I no longer see myself as bad or evil or worthless. It was my misperceptions of events which sent me into a spiral of shame, guilt, and depression.

P12f: Self-esteem in my case is very low but I think that maybe not as bad as it was before I started the program.

In their final impact statements many patients also clearly articulated a cognitive shift in their self-conceptualizations. Patients frequently arrived at a new recognition that they had acted in good conscience during the traumatic event and that, often, elements of the traumatic event were out of their control.

P14f: ... I am becoming aware that I cannot put the blame on myself or others - it happened as it did the results were not truly anything to do with me... I have to accept this and leave the guilt and remorse behind me after 35 years.

\section{Intimacy}

At the beginning of therapy, patients described difficulty with emotional and physical intimacy:

P13i: Whenever I tried to show emotions and make love to my wife... [it] was more or less a forceful situation. I just couldn't let go. 
P12i: I isolate myself to avoid and defuse episodes of anxiety. I go to our land to isolate myself and I come back better able to cope for a while.

Overall, patients reported improvements in intimacy in their final impact statements:

P3f: Although still very much affected, I can feel myself coming out of my shell wanting to participate in life's activities and to be with my family and friends. There is no question, it is time to move on and join the ranks of being a productive nurturing, confident, father, husband and friend.

Despite general improvements in intimacy by the end of therapy, it was common for patients to articulate some degree of continued challenge in demonstrating their feelings of closeness to others.

P5f: [With intimacy I] still struggle, but I think that people know how I feel. I have growing acceptance that people know I have feelings, even if I struggle to show them.

P10f: Intimacy is still a problem. It's hard for me to talk to my wife and we are distant, like I am from my daughter too.

\section{Emotions/symptoms}

In their initial impact statements, many patients referred to the experience of negative affect and the lack of positive affect. Participants frequently mentioned depressive symptoms and PTSD symptoms such as intrusive memories and hypervigilance as well as fears of emotion dysregulation, especially related to anger.

P11i: Things are just not the same anymore. Not interested in things I used to really enjoy.

P15i: I'm always scanning, watching what people are doing. Sometime[s I am] afraid of what I might do if someone touched me and I didn't know they was there. If someone wakes me up, they better stand back. I'll come up swinging...

P5i: I'm afraid if I do let go I won't be able to stop it or control it. It has made me angry with the world so I'm always walking around with a chip on my shoulder waiting for someone to knock it off and also fighting to control it because I'm afraid of what I'd do if not able to stop it.

In final impact statements, several patients described reduced guilt and more frequent experience and expression of emotions, including positive emotions.

P14f: My traumas may always be with me but not... how they have affected me previously. I don't have to isolate or emotionally break down with anxiety and fears.

P6f: I am beginning to really appreciate myself and am realizing that I deserve to feel good inside and to do nice things for myself. I am really allowing myself to trust my current husband and to just relax in being with him and enjoying the moment.

P4f: [This has increased] my ability to experience and describe my happiness as well as all kinds of feelings!!!! I've slowed down so I can enjoy more and appreciate [more].

It was also the case, however, that patients sometimes described negative emotions about their traumatic event despite improvement in other feelings: 
P10f: I have been guilty for years about the death of that boy in Vietnam. I felt it was my fault for not saving him... Now, the guilt about him is gone. I did all that I could when I was there. It was sad that it happened and I am sad when I think about it, but I don't feel guilty.

\section{Perspective on life}

In their initial impact statements, patients commonly described the ways in which their trauma exposure changed their perspective in negative and painful ways:

P3i: The traumatic event(s) that occurred during my tour of duty in Vietnam have impacted virtually every aspect of my life... There is no question, there has been a profound impact on me, ground into the very fiber and fabric of my beliefs and thoughts....

P1i: The shocking trauma that I experienced ... in combat... was the most profound experience of my life. On a very fundamental level, it frightens and disturbs me in a very dark and sinister way ... Understanding it and giving it meaningfulness have become obsessions with me. It subtracts from balance in my life, makes emotional growth difficult and spiritual peace an impossibility. It makes me afraid and ever anxious of the growing imminence of death... It creates a confounding desperation in all that I do... It wears a lot of people out and in the end it will probably wear me out too.

Following treatment, a few patients reiterated the influence their trauma had on their life perspective:

P8f: These two traumatic events that occurred while I was in the service has shattered my belief in myself, others around me and the world.

P13f: It is so hard to understand how someone who wasn't in the war could know what ill affects it caused a soldier. No one can relate to the war... Anyone who went to war bears a harder soul and feels regret and emotional complexes that cannot be described in words.

However, more commonly, patients described a shift in their perspective, often noting a decrease in fear and an increase in well-being and positive affect:

P14f: I sincerely have listened and learned and am slowly implementing these things in my life and it will continue to get so much better thank you with all my heart. This whole new perspective has opened my eyes and ears. I will recognize the truth and trust. I will look at my past as what it was ... it will be a gradual journey into self awareness and a happiness that is awaiting for me.

P1f: Now I can hear my mind, body, emotions, what others have to say because I'm actively listening and I'm getting much better... as a result of it ... I take better care of myself physically, emotionally, psychologically. This has had a very dramatic positive impact on how I go about living my life without dying in the process. It's made me happier. I like who I am, where I am and what I'm doing better now. I'm feeling optimistic in a more peaceful way about my life's growth, development and the possibilities for finding meaning and fulfillment. The awful experience of combat trauma injured my mind and my body and its aftermath stole years of peace and contentment from me but there is no hole in my soul or my song. 


\section{Positive effects of therapy}

There were very few references to therapy in the initial impact statements; however, in their final impact statements, many patients expressed the ways in which therapy made a positive impact on their lives.

P14f: As a result of this study I sincerely believe that I have become acutely aware of my stuck points... I look at situations and do a quick evaluation of them and come out with a more realistic, healthy and positive way of approaching them in a logical manner.

P7f: I can't praise ... [the] therapists involved in this CPT program enough for the help they have given myself and the many other vets like me to get through this.... it is like a new lease on life.

Not a single patient spoke about the treatment in a negative light. However, some patients spoke of the ongoing work of therapy, sometimes describing the process of considering alternative cognitions:

P11f: Although my feelings about my [combat experience] remain largely unchanged, I am now at least willing to consider other ideas. These would be: one, I was not a complete failure. Two, certain events were beyond my control. Three, I do not need forgiveness. Four, I did the best I could in most cases.

Moreover, patients often described gratitude and hopefulness about the future while remaining grounded in the realistic view that their trauma would likely continue to have an impact on their lives.

\section{Discussion}

This study examined patients' appraisals of their traumatic experiences and trauma-related beliefs to better understand the outcomes and mechanisms of CPT. Employing thematic analysis to examine changes in language use provided a more in-depth and nuanced understanding of cognitive changes occurring over the course of therapy. As predicted, we found that patients included fewer maladaptive statements and more adaptive statements in the final impact statement, reflecting the CPT themes of safety, trust, power/control, esteem, and intimacy. Patients included fewer references to PTSD symptoms and related negative emotions (e.g. guilt, fear, and anger) in final impact statements relative to initial impact statements. There was a shift in perspective following treatment, with patients moving from a negative perspective of the trauma to a more positive perspective. These changes in perspective were of particular interest in the current study due to the proposed cognitive mechanisms of CPT. These findings support the idea that the focus on changing maladaptive cognitions about patients' traumatic experiences has an impact on how patients view their experience.

Although patients tended to note improvement in negative emotions in their final impact statements, there were some exceptions. Some individuals described continued sadness about aspects of their traumatic experience. This finding is consistent with a cognitive conceptualization of PTSD positing that, although patients typically experience a reduction in negative emotions, some negative experiences or emotions may remain, and individuals will 
typically be better able to tolerate and express those negative emotions after treatment. Many individuals with PTSD report high levels of experiential avoidance, and improvements may be mediated by the greater experience, expression, and tolerance of all emotions, including negative ones (Walser and Hayes, 2006).

Thematic analysis suggested that CPT may increase the experience and expression of positive emotions. Several patients acknowledged greater happiness and other positive emotions in final impact statements, and many patients described positive responses to the therapy overall. This is consistent with other qualitative studies of trauma-focused CBT that have highlighted patients' positive experiences with trauma-focused therapy, despite initial ambivalence or anxiety about beginning treatment (e.g. Shearing et al., 2011).

Moreover, our findings suggest that patients benefited from CPT in a variety of ways. Consistent with other recent qualitative investigations of CPT (Sobel et al., 2009) as well as clinical observation that individuals often describe personal changes following therapy that may not be documented in traditional psychometric outcome measures, our findings further support using qualitative methods in order to capture these important outcomes of psychotherapy.

One particularly beneficial aspect of the current study's use of impact statements to examine response to CPT is that these statements are not intended as measures of patient satisfaction. Yet, patients often used them as ways to communicate about the benefits of therapy. This indirect approach to investigating treatment response minimizes concerns about artificially inflated self-reported measures of improvement.

Limitations of our study include our small sample and the fact that our writing samples were somewhat thematically guided due to the nature of the impact statement assignment (i.e. the statement content was not unstructured or spontaneous). Also, our participants were military veterans, and this characteristic of our sample may limit the generalizability of our findings to other patient populations. Further, our sample included only those patients who had both initial and final impact statements available; it is possible that these patients differed from patients who did not complete the final impact statement. Although coders were blind to whether statements were initial or final statements and we utilized codes that supported objectivity, it is possible that coders may have had unintentional biases that may have influenced their interpretations, particularly in instances where unblinding occurred (e.g. when a patient referenced the benefits of treatment). Although our findings are consistent with the proposed cognitive mechanisms of CPT, this study does not establish causal associations between cognitive changes and PTSD outcomes. An alternative explanation of these findings is that the writing and reading of written accounts in this application of CPT played a role in these changes in light of research demonstrating cognitive changes in exposure-based treatments (Foa and Rauch, 2004). Future similar research with the cognitive-only version of CPT would help shed light on this possibility.

In conclusion, the current study suggests that incorporating thematic analysis into therapy outcome research can aid in assessing the extent to which cognitive changes, such as increases in insight and changes in beliefs about the self, others, and the world, mediate improvements in psychological symptoms following CPT. It also helps clinicians and researchers better understand the types of changes to be expected with cognitive therapies for PTSD, which hopefully will facilitate more effective therapy delivery. 


\section{References}

Blake, D., Weathers, F., Nagy, L., Kaloupek, D., Gusman, F. D., Charney, D., et al. (1995). The development of a clinician-administered PTSD scale. Journal of Traumatic Stress, 8, 75-90. doi: $10.1002 /$ jts. 2490080106

Chard, K. (2005). An evaluation of cognitive processing therapy for the treatment of posttraumatic stress disorder related to childhood sexual abuse. Journal of Consulting and Clinical Psychology, 73, 965-971. doi: 10.1037/0022-006X.73.5.965

Foa, E. B. and Rauch, S. A. M. (2004). Cognitive changes during prolonged exposure versus prolonged exposure plus cognitive restructuring in female assault survivors with posttraumatic stress disorder. Journal of Consulting and Clinical Psychology, 72, 879-884. doi: 10.1037/0022-006X.72.5.879

Gallegos, N. (2005). Client perspectives on what contributes to symptom relief in psychotherapy: a qualitative outcome study. Journal of Humanistic Psychology, 45, 355-382. doi: $10.1177 / 0022167805277106$

Klein, M. and Elliott, R. (2006). Client accounts of personal change in process-experiential psychotherapy: a methodologically pluralistic approach. Psychotherapy Research, 16, 91-105. doi: 10.1080/10503300500090993

Klest, B. and Freyd, J. (2007). Global ratings of essays about trauma: development of the GREAT code, and correlations with physical and mental health outcomes. Journal of Psychological Trauma, 6, 1-20. doi: 10.1300/J513v06n01_01

Monson, C., Schnurr, P., Resick, P., Friedman, M., Young-Xu, Y. and Stevens, S. (2006). Cognitive Processing Therapy for veterans with military-related posttraumatic stress disorder. Journal of Consulting and Clinical Psychology, 74, 898-907. doi: 10.1037/0022-006X.74.5.898

O'Kearney, R. and Perrott, K. (2006). Trauma narratives in posttraumatic stress disorder: a review. Journal of Traumatic Stress, 19, 81-93. doi: 10.1002/jts.20099

Pennebaker, J., Booth, R. and Francis, M. (2007). Linguistic Inquiry and Word Count: LIWC 2007. Austin, TX: LIWC (www.liwc.net).

Resick, P., Galovski, T., Uhlmansiek, M., Scher, C., Clum, G. and Young-Xu, Y. (2008). A randomized clinical trial to dismantle components of cognitive processing therapy for posttraumatic stress disorder in female victims of interpersonal violence. Journal of Consulting and Clinical Psychology, 76, 243-258. doi: 10.1037/0022-006X.76.2.243

Resick, P., Monson, C. and Chard, K. (2007). Cognitive Processing Therapy: veteran/military version. Washington, DC: Department of Veterans' Affairs.

Shearing, V., Lee, D. and Clohessy, S. (2011). How do clients experience reliving as part of traumafocused cognitive behavioural therapy for posttraumatic stress disorder? Psychology and Psychotherapy: Theory, Research and Practice, 84, 458-472. doi: 10.1111/j.2044-8341.2010.02012.x

Sobel, A., Resick, P. and Rabalais, A. (2009). The effect of cognitive processing therapy on cognitions: impact statement coding. Journal of Traumatic Stress, 22, 205-211. doi: 10.1002/jts.20408

Stone, P. (1997). Thematic text analysis: new agendas for analyzing text content. In C. Roberts (Ed)., Text Analysis for the Social Sciences. Mahwah, NJ: Lawrence Erlbaum Associates.

Tausczik, Y. R. and Pennebaker, J. W. (2010). The psychological meaning of words: LIWC and computerized text analysis methods. Journal of Language and Social Psychology, 29, 24-54. doi: $10.1177 / 0261927 \times 09351676$

Vincent, F., Jenkins, H., Larkin, M., and Clohessy, S. (2012). Asylum seekers' experiences of trauma focused cognitive behaviour therapy for posttraumatic stress disorder: a qualitative study. Behavioural and Cognitive Psychotherapy, 41, 579-593. doi: 10.1017/S1352465812000550

Walser, R. and Hayes, S. (2006). Acceptance and commitment therapy and trauma survivors. In V. Follette (Ed.), Trauma in Context: a cognitive behavioral approach to trauma (2nd ed.) New York: Guilford Press. 